\title{
Effect of Methadone on Cardiac Repolarization in Japanese Cancer Patients: A Longitudinal Study
}

Eriko Yamanaka - Satoru Chino - Toshifumi Takasusuki ·

Shinsuke Hamaguchi · Shigeki Yamaguchi

Received: September 11, 2019 / Published online: November 20, 2019

(C) The Author(s) 2019

\section{ABSTRACT}

Introduction: Methadone is known to prolong the QT interval, which could induce lethal arrhythmias such as torsades de pointes. To determine the risk of ventricular arrhythmias in cancer patients using methadone, we measured QT dispersion (QTD) and Tpeak-Tend (TpTe) before and after methadone administration and evaluated the correlations between methadone dosage and cardiac repolarization.

Methods: We conducted a retrospective observational study with 19 patients undergoing follow-up for cancer pain with methadone. Electrocardiogram (ECG) recordings were obtained from the patients at methadone initiation and 1 week, 1 month, and 2 months later. The QT, corrected QT (QTc), QTD, QTc dispersion (QTcD), TpTe, TpTe/QT, and TpTe/QTc

Enhanced Digital Features To view enhanced digital features for this article go to https://doi.org/10.6084/ m9.figshare.10059275.

E. Yamanaka $\cdot$ S. Chino $\cdot$ T. Takasusuki $(\bowtie)$.

S. Hamaguchi · S. Yamaguchi

Department of Anesthesiology, School of Medicine,

Dokkyo Medical University, Kitakobayashi 880,

Mibu, Tochigi 321-0293, Japan

e-mail: takasusu@dokkyomed.ac.jp were measured manually via ECG records and analyzed using a repeated measures one-way ANOVA. The correlations between these ECG parameters and each methadone dosage were determined using Spearman's rank correlation coefficient.

Results: The QTD, QTcD, TpTe/QT, and TpTe/ QTc remained unchanged, while TpTe was prolonged significantly at 2 months (initiation: $82 \pm 17$ ms; 2 months: $106 \pm 20 \mathrm{~ms}, p=0.018$ ). In addition, there was a positive correlation between TpTe and methadone dosage ( $\mathrm{rs}=0.4$, $p=0.041$ ).

Conclusions: The findings suggested that small or modest doses of methadone could exert dosedependent effects on cardiac repolarization in cancer patients.

Trial Registration: UMIN Clinical Trials Registry, UMIN000034519.

Keywords: Cardiac repolarization; Methadone; QT dispersion; QT interval; T peak-T end 


\section{Key Summary Points}

Why Carry Out This Study?

Methadone is known to induce QT prolongation, which can lead to torsade de pointes. However, several studies suggested that methadone does not prolong the QT interval.

To clarify whether methadone induces arrhythmia in cancer patients, we evaluated cardiac repolarization using Tpeak-Tend, which is considered to be an index of ventricular arrhythmia.

What Was Learned from the Study?

Small or modest doses of methadone significantly prolonged Tpeak-Tend 2 months after initiation of methadone administration.

Careful attention should be paid to patients receiving methadone. TpeakTend can be a marker for increased risk of lethal arrhythmia in these patients.

\section{INTRODUCTION}

The synthetic opioid methadone has unique pharmacokinetic properties including those of long-acting $\mu$ and $\delta$ opioid receptor agonists, an $\mathrm{N}$-methyl-D-aspartate (NMDA) receptor antagonist, and inhibition of reuptake of serotonin and norepinephrine [1]. Because of these unique properties, this analgesic contributes to the relief of severe chronic pain that is refractory to other opioids for long periods. Methadone was recently approved in Japan for use only for severe cancer pain refractory to other opioids. However, it could induce several adverse effects, such as prolongation of the QT interval, which may represent a risk factor for the occurrence of torsades de pointes (TdP) [2]. Methadone has been identified as the secondmost common suspected cause of drug-induced corrected QT (QTC) prolongation or torsade de pointes since 2000 in the USA [3]. Therefore, routine close electrocardiogram (ECG) observation is recommended for methadone maintenance therapy.

The QT dispersion (QTD) is defined as the difference between maximal and minimal QT intervals on a 12-lead surface ECG and reflects the regional heterogeneity of ventricular repolarization. Further, it has been regarded as an index of ventricular arrhythmia, which may lead to sudden cardiac death [4]. Moreover, methadone has been reported to increase QTD in patients with opioid dependency [5].

The prolongation of the peak and end of the $\mathrm{T}$ wave interval (TpTe) in the 12-lead ECG, which could reflect the transmural dispersion of repolarization (TDR), has been associated with the incidence of ventricular arrhythmias $[6,7]$. Similar to TpTe, the TpTe/QT ratio is considered as a crucial marker of TDR and a noninvasive arrhythmogenic index of sudden cardiac death [6]. A previous study reported that TpTe was prolonged by methadone [8]. However, it is important to note that most previous studies involved patients with opioid dependency but not cancer. Therefore, our hypothesis was that methadone would affect cardiac repolarization, which is determined by the QTD and TpTe in cancer patients, in a dose-dependent manner. To determine the risk of ventricular arrhythmias in cancer patients using methadone, we evaluated the change in QTD and TpTe before and after administration of methadone.

\section{METHODS}

The study was approved by the ethics committee at Dokkyo Medical University (registration number: R-12-16J) and registered at the UMIN Clinical Trials Registry (registration number: UMIN000034519). All procedures performed in studies involving human participants were in accordance with the ethical standards of the institutional and national research committee and the 1964 Helsinki Declaration and its later amendments or comparable ethical standards. Informed consent was obtained from all individual participants included in the study.

To clarify the effects of small-to-modest doses of methadone on cardiac repolarization in 
cancer patients, we measured the QTD, QTc dispersion (QTcD), TpTe, TpTe/QT, and TpTe/ QTc using a 12-lead ECG. In addition, we analyzed the correlations between ECG parameters and methadone dosage.

We conducted a retrospective review of medical records of 19 patients aged 29-85 years who were treated with methadone for cancer pain at Dokkyo Medical Hospital between April 2013 and March 2019. Informed consent for use of patient's data was obtained from all participants at the hospital admission. Participants with cardiovascular, respiratory, or cerebrovascular disease; ECG abnormalities; or other types of medication that could lead to QT prolongation were excluded from the study. At the initiation of methadone administration, standard 12-lead ECG recordings were obtained from patients at a paper speed of $25 \mathrm{~mm} / \mathrm{s}$. The QT interval, QTc interval, QTD, QTcD, TpTe interval, TpTe/QT ratio, and TpTe/QTc ratio were measured manually via ECG recordings, which were obtained 1 week, 1 month, and 2 months after initiation of methadone administration and compared. The QT interval was measured using the tangent method. It was defined as the interval from the onset of the $\mathrm{Q}$ wave, which was defined as the intersection of a threshold level with the differential of the Q wave, to the end of the $\mathrm{T}$ wave, which was defined as the intersection of a tangent to the steepest slope of the last limb of the $\mathrm{T}$ wave and the baseline in leads II and V5. The QT interval was corrected using Fridericia's formula $(\mathrm{QTc}=\mathrm{QT} / 3 \sqrt{ } \mathrm{RR})$. The TpTe interval was defined as the interval from the peak of the $\mathrm{T}$ wave to the end of the $\mathrm{T}$ wave in leads II and V5. Similar to the QT measurement, the end of the $T$ wave was determined using the tangent method. Three consecutive cycles in leads II and V5 were measured and averaged to analyze the QT and TpTe intervals. QTD was defined as the difference between the maximum and minimum mean QT intervals on the 12-lead ECG. The mean value for data derived from three successive beats for each lead was used in the analysis. Leads for which the end of the $T$ wave in 12-lead ECGs could not be clearly detected were excluded from the analysis.

\section{Statistical Analysis}

Data are expressed as means \pm standard deviations. The QT interval, QTc interval, QTD, QTcD, TpTe interval, TpTe/QT ratio, and TpTe/ QTc ratio were analyzed using repeated measures one-way analysis of variance. When a significant overall effect was detected, Dunnett's post hoc test was performed. Correlations between the ECG parameters and methadone dosages were analyzed using Spearman's rank correlation coefficient. All statistical analyses were performed using Prism 6 (GraphPad, San Diego, CA, USA). In all analyses, $p$ values of $<$ 0.05 were considered significant.

\section{RESULTS}

Following the screening of medical records, one patient was excluded from the study because of an indistinct $\mathrm{T}$ wave in 12-lead ECGs. Additionally, one patient was excluded due to ischemic heart disease. Table 1 shows the participants' characteristics including chemotherapy and radiation therapy the patients received. No cardiac complications were observed throughout the study period.

Table 2 shows the mean methadone dose and values for the QT interval, QTc interval, QTD, QTcD, TpTe, TpTe/QT ratio, and TpTe/ QTc ratio before and after the administration of methadone. The mean methadone dose gradually increased during the observation period (1 week: $20 \pm 7 \mathrm{mg} ; 1$ month: $36 \pm 13 \mathrm{mg}$, 2 months: $53 \pm 23 \mathrm{mg}$ ). The QT and QTc were within the normal range in all participants before the administration of methadone (QT < 0.47 , male: QTc $<0.44$, female: QTc $<0.46$ ). However, prolongation of QTc was seen in 2 patients (12\%) at 1 and 2 months, and in 4 patients (24\%) at 2 months after administration of methadone, respectively. The TpTe in lead II had increased significantly 2 months after initiation of methadone (baseline: $82 \pm 17 \mathrm{~ms}$; 2 months: $106 \pm 20 \mathrm{~ms}, p=0.018)$. Similarly, TpTe in lead V5 had significantly increased 2 mounts after initiation of methadone (baseline: $81 \pm 17 \mathrm{~ms} ; \quad 2$ months: $\quad 107 \pm 18 \mathrm{~ms}$, 
Table 1 Patient characteristics

\begin{tabular}{|c|c|}
\hline Sex (male/female) & $5 / 12$ \\
\hline Age (years) & $56.1 \pm 16.0$ \\
\hline Primary oncological diagnosis & $n$ \\
\hline Colon cancer & 5 \\
\hline Lung cancer & 3 \\
\hline Breast cancer & 2 \\
\hline Laryngeal cancer & 2 \\
\hline Multiple myeloma & 1 \\
\hline Pharyngeal cancer & 1 \\
\hline Uterine cancer & 1 \\
\hline Chordoma & 1 \\
\hline External auditory canal cancer & 1 \\
\hline \multicolumn{2}{|l|}{ Medication } \\
\hline Hypertension & 5 \\
\hline Hyperlipidemia & 2 \\
\hline Diabetes mellitus & 2 \\
\hline \multicolumn{2}{|l|}{ Chemotherapeutic agents } \\
\hline Carboplatin & 5 \\
\hline 5-Fluorouracil & 3 \\
\hline Paclitaxel & 3 \\
\hline Adriamycin & 2 \\
\hline Cisplatin & 2 \\
\hline Docetaxel hydrate & 2 \\
\hline Melphalan & 2 \\
\hline Pemetrexed sodium hydrate & 2 \\
\hline Prednisolone & 2 \\
\hline Bortezomib & 1 \\
\hline Cetuximab & 1 \\
\hline Doxorubicin hydrochloride & 1 \\
\hline Gimeracil & 1 \\
\hline Ifosfamide & 1 \\
\hline Levofolinate calcium & 1 \\
\hline Oteracil & 1 \\
\hline
\end{tabular}

Table 1 continued

\begin{tabular}{ll}
\hline Oxaliplatin & 1 \\
Tegafur & 1 \\
Radiation therapy & 7 \\
\hline
\end{tabular}

$p=0.0067)$. All other parameters, such as QT, QTc, QTD, QTcD, TpTe/QT, and TpTe/QTc, increased slightly; however, no significant differences were observed.

Figure 1 shows the correlations between the ECG parameters and each methadone dose. The correlations between ECG parameters and methadone dosage were analyzed for each increase in methadone dosage but not for individual patients. The TpTe was positively correlated with methadone dose ( $\mathrm{rs}=0.4$, $p=0.041$; Fig. 1e). However, there were no positive correlations between QT $(\mathrm{rs}=0.27)$, QTc (rs = 0.13), QTD ( $\mathrm{rs}=0.01)$, QTcD, ( $\mathrm{rs}=$ 0.03), TpTe/QT ( $r s=0.25$ ), or TpTe/QTc ( $r s=$ 0.13) and methadone dose (Fig. 1a-d, f, g).

\section{DISCUSSION}

\section{Methadone on QT Interval}

It is known that methadone, a long-acting synthetic opioid, prolongs the QTc interval in patients with opioid addiction [9]. One previous study suggested that a QTc of $>500 \mathrm{~ms}$ was observed in $2 \%$ of patients who used $>100 \mathrm{mg}$ of methadone per day, and sudden cardiac death associated with methadone use has been reported at a dose of $29 \mathrm{mg}$ per day [10]. These findings demonstrate that lethal arrhythmias could occur within a wide dose range. In contrast, one study indicated that significant QT prolongation was infrequent in pediatric patients with cancer [11]. The safety of prescribing methadone to cancer patients remains unclear. If the QT interval is within $450-500 \mathrm{~ms}$, methadone may be initiated or continued with frequent monitoring. For methadone-maintained patients with QT prolongation more than $500 \mathrm{~ms}$, a risk minimization strategy should be strongly considered [12]. 
Table 2 Dose of methadone and electrocardiographic variables before and after treatment

\begin{tabular}{llccr}
\hline & Baseline & 1 week & 1 month & 2 months \\
\hline Mean dose of methadone (mg) & 0 & $20 \pm 7$ & $36 \pm 13$ & $53 \pm 23$ \\
QT in II (ms) & $349 \pm 46$ & $356 \pm 32$ & $390 \pm 44$ & $379 \pm 50$ \\
QT in V5 (ms) & $338 \pm 49$ & $348 \pm 35$ & $361 \pm 62$ & $365 \pm 52$ \\
QTc in II (ms) & $392 \pm 31$ & $408 \pm 39$ & $410 \pm 40$ & $425 \pm 42$ \\
QTc in V5 (ms) & $393 \pm 42$ & $399 \pm 34$ & $411 \pm 37$ & $412 \pm 27$ \\
QTD (ms) & $58 \pm 17$ & $77 \pm 28$ & $72 \pm 36$ & $82 \pm 29$ \\
QTcD (ms) & $69 \pm 23$ & $88 \pm 33$ & $94 \pm 40$ & $97 \pm 41$ \\
TpTe in II (ms) & $82 \pm 17$ & $84 \pm 10$ & $98 \pm 17$ & $106 \pm 20^{*}$ \\
TpTe in V5 (ms) & $80 \pm 16$ & $83 \pm 12$ & $92 \pm 16$ & $107 \pm 18^{*}$ \\
TpTe/QT in II & $0.24 \pm 0.07$ & $0.23 \pm 0.03$ & $0.25 \pm 0.06$ & $0.25 \pm 0.06$ \\
TpTe/QT in V5 & $0.24 \pm 0.06$ & $0.24 \pm 0.04$ & $0.24 \pm 0.04$ & $0.26 \pm 0.04$ \\
TpTe/QTc in II & $0.20 \pm 0.04$ & $0.2 \pm 0.03$ & $0.22 \pm 0.06$ & $0.21 \pm 0.04$ \\
TpTe/QTc in V5 & $0.20 \pm 0.03$ & $0.21 \pm 0.02$ & $0.21 \pm 0.03$ & $0.23 \pm 0.02$ \\
\hline
\end{tabular}

Data are presented as means \pm standard deviations

QTD QT dispersion, QTcD QTc dispersion, $T p T e \mathrm{~T}$ peak-T end

${ }^{*} p<0.05$ versus baseline value

In the current study, QT and QTc were slightly, but not significantly, prolonged. The current results suggest that low doses of methadone might not significantly prolong the QT or QTc.

\section{Effects of Methadone on Cardiac Repolarization}

Several studies have shown that increased QTD was associated with arrhythmogenicity. QTD and the QTD ratio (divided by cycle length and expressed as a percentage) in patients with acute myocardial infarction exhibiting ventricular fibrillation were significantly higher relative to those observed in patients with unstable angina [13]. It has been reported that methadone led to modest increases in QTD and TpTe $[5,8]$. The TpTe was associated with increased mortality (a cut-off value of $100 \mathrm{~ms}$ ) [14]. Moreover, prolongation of the TpTe interval was independently associated with sudden cardiac death when the QTc was normal or not measurable [15]. In the present study, methadone prolonged TpTe, but not QTD, 2 months after treatment initiation (>100 ms). Generally, QTD indicates the spatial dispersion of cardiac repolarization, whereas TpTe expresses the transmural dispersion of repolarization. Previous study indicated that TpTe could reflect local repolarization [16]. Although the interpretation of these conflicting results remains uncertain, we hypothesize that these results arise owing to the following reasons: (1) sample size was inadequate to detect the spatial dispersion of cardiac repolarization; (2) we did not have multiple TpTe measurements that are required to detect the accurate transmural inhomogeneity; (3) TpTe was more easily influenced by QT prolongation than QTD. In terms of QT prolongation, TpTe/QT or TpTe/QTc may be more sensitive than TpTe. The present study emphasizes that even low doses of methadone might prolong the TpTe. Therefore, the possibility of arrhythmogenicity during the treatment of cancer pain using methadone should be considered. 
A

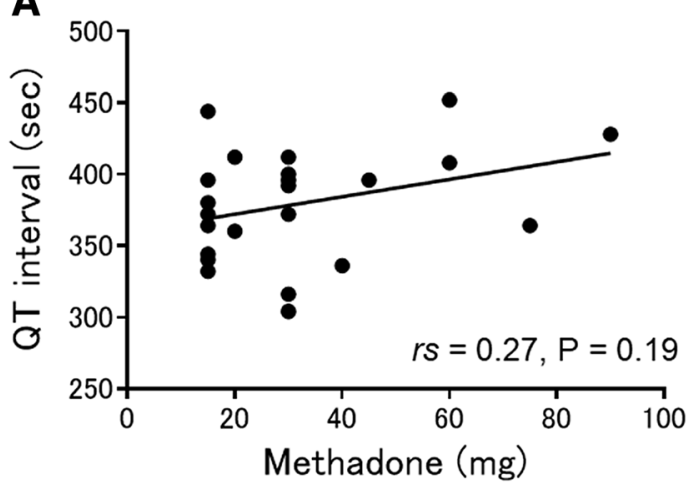

C

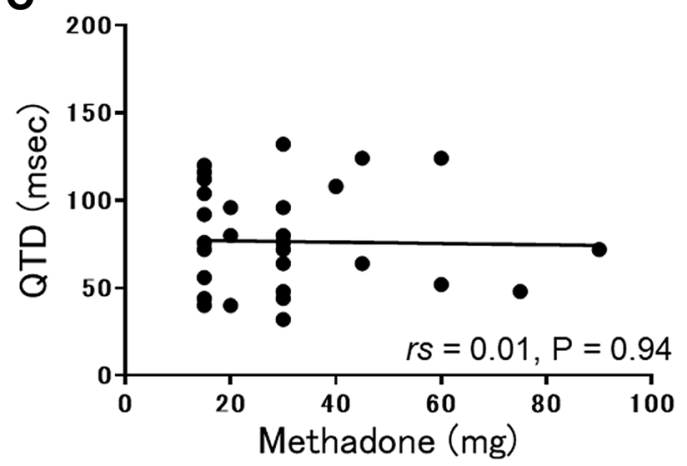

E

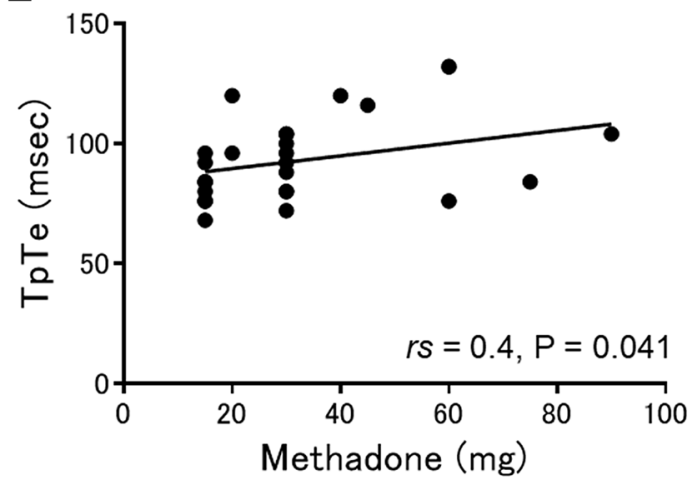

B

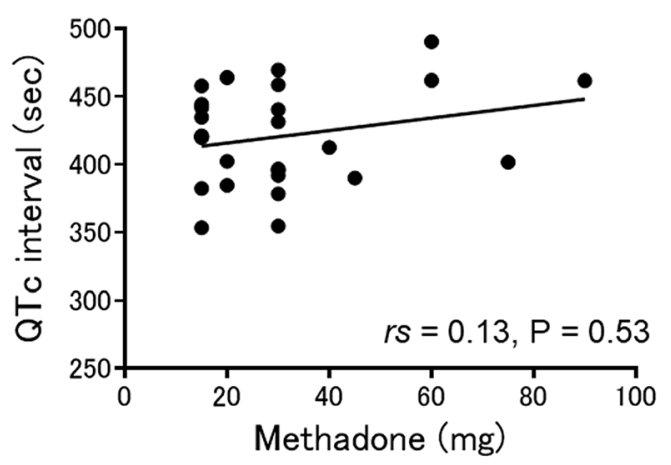

D

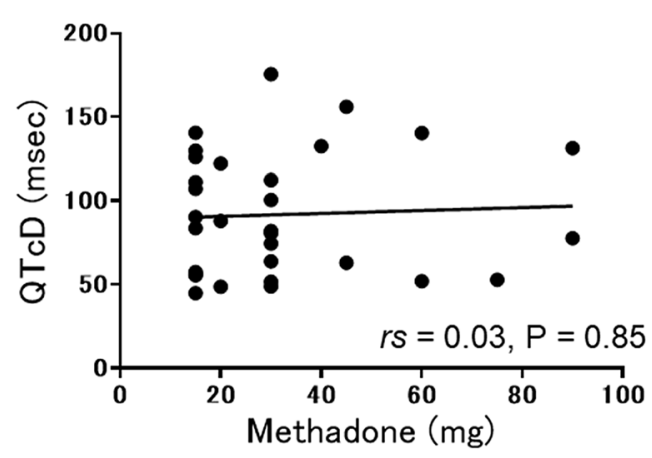

F

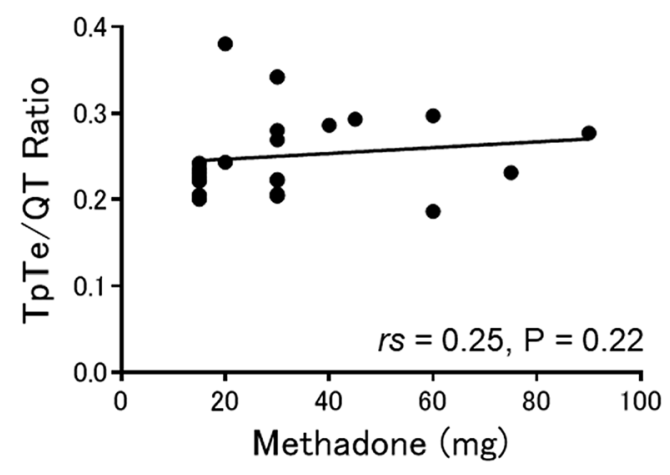

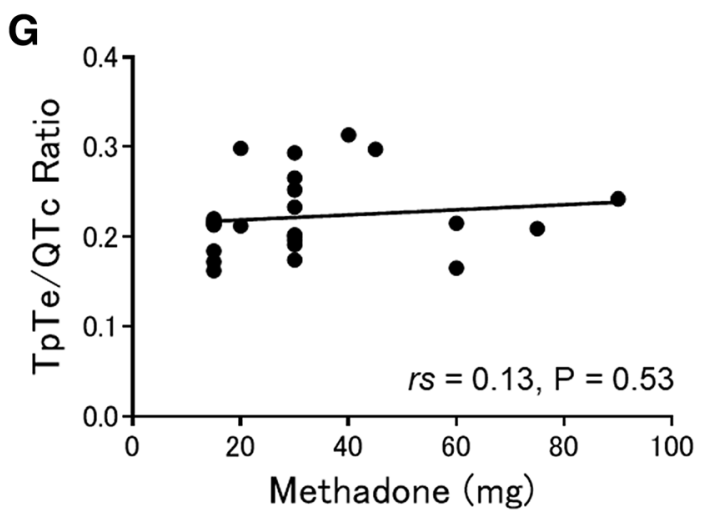


4Fig. 1 Correlations between methadone doses and ECG parameters. a-d, f, $\mathbf{g}$ There were no positive correlations between QT interval ( $\mathrm{rs}=0.27$ ), QTc interval ( $\mathrm{rs}=0.13$ ), QTD $\quad(\mathrm{rs}=0.01), \quad \mathrm{QTcD} \quad(\mathrm{rs}=0.03), \quad \mathrm{TpTe} / \mathrm{QT}$ (rs $=0.25)$, or $\mathrm{TpTe} / \mathrm{QTc}(\mathrm{rs}=0.13)$ and methadone dosage. e In contrast, there was a positive correlation between $\mathrm{TpTe}$ and methadone dosage $(\mathrm{rs}=0.4$, $p=0.041$ )

There was no significant correlation between QT, QTc, QTD, or QTcD, and methadone dose. With respect to $\mathrm{TpTe}$, there was no significant correlation between TpTe/QT or TpTe/QTc and methadone dose. In contrast, TpTe was significantly correlated with each dose of methadone. These contradictions might arise owing to the inadequate sample size of this study. Further study is essential for evaluating the correlation between cardiac repolarization and methadone dose. We suggest that the consideration should be given to discontinue the methadone-maintained patients with TpTe more than 100 ms.

\section{Limitations}

Methadone has been approved in Japan since 2013. It is a comparatively novel opiate, therefore there is limited knowledge regarding the use of methadone in Japanese cancer patients. In the current explorative study, only a small sample of 19 patients was available. Unlike in other countries, there is no established treatment for opioid addiction. Moreover, the use of methadone is still restricted to refractory cancer pain in Japan. Therefore, the consumption of methadone did not increase compared to other countries. In addition, this study was a singlecenter, retrospective, observational study. To clarify the influence of methadone on cardiac repolarization, further analysis via multi-center prospective studies is essential.

It has been suggested that some cancer types may affect the QT interval. In the univariate logistic regression analysis, breast cancer and gastrointestinal cancer and their supportive drugs could affect QT prolongation [17]. It is likely that the QT interval was affected by the peculiarity of cancer type in several cases in our study.

\section{CONCLUSIONS}

The QT, QTc, QTD, QTcD, TpTe/QT, and TpTe/ QTc were not prolonged, while TpTe was prolonged in a dose-dependent manner after methadone. Although the study question remains unanswered, this latter result suggests that methadone might increase the risk of ventricular arrhythmia in a dose-dependent manner.

\section{ACKNOWLEDGEMENTS}

We thank the participants of the study.

Funding. No funding or sponsorship was received for this study or publication of this article. The Rapid Service Fee was funded by the authors.

Medical Writing, Editorial, and Other Assistance. Writing assistance in the preparation of this article was provided by Editage (http://www.editage.jp). Financial support for this assistance was provided by the authors.

Authorship. All named authors meet the International Committee of Medical Journal Editors (ICMJE) criteria for authorship for this article, take responsibility for the integrity of the work as a whole, and have given their approval for this version to be published.

Disclosures. Eriko Yamanaka, Satoru Chino, Toshifumi Takasusuki, Shinsuke Hamaguchi and Shigeki Yamaguchi have nothing to disclose.

Compliance with Ethics Guidelines. The study was approved by the ethics committee at Dokkyo Medical University (registration number: R-12-16J) and registered at the UMIN Clinical Trials Registry (registration number: UMIN000034519). All procedures performed in studies involving human participants were in 
accordance with the ethical standards of the institutional and national research committee and the 1964 Helsinki Declaration and its later amendments or comparable ethical standards. Informed consent was obtained from all individual participants included in the study.

Data Availability. The datasets generated during and/or analyzed during the current study are available from the corresponding author on reasonable request.

Open Access. This article is distributed under the terms of the Creative Commons Attribution-NonCommercial 4.0 International License (http://creativecommons.org/licenses/ by-nc/4.0/), which permits any noncommercial use, distribution, and reproduction in any medium, provided you give appropriate credit to the original author(s) and the source, provide a link to the Creative Commons license, and indicate if changes were made.

\section{REFERENCES}

1. Davis MP, Walsh D. Methadone for relief of cancer pain: a review of pharmacokinetics, pharmacodynamics, drug interactions and protocols of administration. Support Care Cancer. 2001;9:73-83.

2. Pearson EC, Woosley RL. QT prolongation and torsades de pointes among methadone users: reports to the FDA spontaneous reporting system. Pharmacoepidemiol Drug Saf. 2005;14:747-53.

3. Kao D, Bucher Bartelson B, Khatri V, et al. Trends in reporting methadone-associated cardiac arrhythmia, 1997-2011: an analysis of registry data. Ann Intern Med. 2013;158:735-40.

4. Tse G, Yan BP. Traditional and novel electrocardiographic conduction and repolarization markers of sudden cardiac death. Europace. 2017;19:712-21.

5. Krantz MJ, Lowery CM, Martell BA, Gourevitch MN, Arnsten JH. Effects of methadone on QT-interval dispersion. Pharmacotherapy. 2005;25:1523-9.

6. Gupta P, Patel C, Patel H, et al. T(p-e)/QT ratio as an index of arrhythmogenesis. J Electrocardiol. 2008;41:567-74.
7. Kors JA, van Ritsema HJ, van Herpen G. The meaning of the Tp-Te interval and its diagnostic value. J Electrocardiol. 2008;41:575-80.

8. Acciavatti T, Martinotti G, Corbo M, et al. Psychotropic drugs and ventricular repolarisation: the effects on QT interval, T-peak to T-end interval and QT dispersion. J Psychopharmacol. 2017;31:453-60.

9. Martell BA, Arnsten JH, Krantz MJ, Gourevitch MN. Impact of methadone treatment on cardiac repolarization and conduction in opioid users. Am J Cardiol. 2005;95:915-8.

10. Krantz MJ, Martin J, Stimmel B, Mehta D, Haigney MC. QTc interval screening in methadone treatment. Ann Intern Med. 2009;150:387-95.

11. Madden K, Park M, Liu D, Bruera E. The frequency of QTc prolongation among pediatric and young adult patients receiving methadone for cancer pain. Pediatr Blood Cancer. 2017;64:26614.

12. Martin JA, Campbell A, Killip T, et al. QT interval screening in methadone maintenance treatment: report of a SAMHSA expert panel. J Addict Dis. 2011;30:283-306.

13. Higham PD, Furniss SS, Campbell RW. QT dispersion and components of the QT interval in ischaemia and infarction. Br Heart J. 1995;73:32-6.

14. Haarmark C, Hansen PR, Vedel-Larsen E, et al. The prognostic value of the Tpeak-Tend interval in patients undergoing primary percutaneous coronary intervention for ST-segment elevation myocardial infarction. J Electrocardiol. 2009;42:555-60.

15. Panikkath R, Reinier K, Uy-Evanado A, et al. Prolonged Tpeak-to-tend interval on the resting ECG is associated with increased risk of sudden cardiac death. Circ Arrhythm Electrophysiol. 2011;4:441-7.

16. Patel C, Burke JF, Patel H, et al. Is there a significant transmural gradient in repolarization time in the intact heart? Cellular basis of the $\mathrm{T}$ wave: a century of controversy. Circ Arrhythm Electrophysiol. 2009;2:80-8.

17. Khan Q, Ismail $M$, Khan S. Frequency, characteristics and risk factors of QT interval prolonging drugs and drug-drug interactions in cancer patients: a multicenter study. BMC Pharmacol Toxicol. 2017;18:75. 